\title{
OPEN Neutralizing antibodies against SARS-CoV-2 variants of concern elicited by the comirnaty COVID-19 vaccine in nursing home residents
}

\begin{abstract}
Beatriz Sánchez-Sendra ${ }^{1}$, Eliseo Albert ${ }^{2}$, Joao Zulaica ${ }^{1}$, Ignacio Torres ${ }^{2}$, Estela Giménez ${ }^{2}$, Pilar Botija ${ }^{3}$, María José Beltrán ${ }^{4}$, Celia Rodado ${ }^{5}$, Ron Geller ${ }^{1,7}$ \& David Navarro $2,6,7 \bowtie$

Immunosenescence may impact the functionality and breadth of vaccine-elicited humoral immune responses. The ability of sera to neutralize the SARS-CoV-2 spike protein (S) from Beta, Gamma, Delta, and Epsilon variants of concern (VOCs) relative to the ancestral Wuhan-Hu-1 strain was compared in Comirnaty COVID-19-vaccinated elderly nursing home residents, either SARS-CoV-2 naïve $(n=22)$ or experienced $(n=8)$, or SARS-CoV-2 naïve younger individuals $(n=18)$ and non-vaccinated individuals who recovered from severe COVID-19 $(n=19)$. In all groups, except that including SARS-CoV-2experienced nursing home residents, some participants lacked NtAb against one or more VOCs, mainly the Beta variant (15-20\%). Serum NtAb titers were lowest against the Beta variant followed by Gamma, Delta and Epsilon variants. Overall, fold change reduction in NtAb titers relative to the ancestral strain was greatest for the Beta variant (6.7-19.4) followed by Gamma (4.8-16.0), Epsilon (2.9-13.4), and Delta (3.5-6.5) variants, although subtle differences were observed for Beta, Epsilon and Delta variants across comparison groups. In summary, older age, frailty, and concurrence of co-morbidities had no major impact on the serum NtAb activity profile against SARS-CoV-2 VOCs.
\end{abstract}

Coronaviruses encode a proof-reading mechanism to minimize sequence variation in the long RNA genomes that may compromise their survival in nature ${ }^{1}$. Nevertheless, generation of variants during coronavirus replication in humans unfailingly ensues ${ }^{2}$. Many SARS-CoV-2 variants have emerged since its appearance late in 2019, some of which may pose a threat to pandemic control due to their relative resistance to neutralizing activity of antibodies elicited following natural infection or vaccination with the original SARS-CoV-2 isolate (Wuhan/ $\mathrm{Hu}-1$ ); these variants, termed variants of concern (VOC), incorporate amino acid substitutions or deletions in critical locations within the virus Spike (S) protein ${ }^{3}$. The Comirnaty mRNA COVID-19 vaccine encodes the ancestral strain $S$ protein in a pre-fusion conformation ${ }^{4}$. Several studies have shown a decreased ability of serum antibodies elicited by this vaccine to neutralize a number of VOCs compared to the original strain, most notably Beta (B.1.351), Gamma (P.1), and more recently Delta (B.1.617.2) $)^{5-12}$. Nevertheless, elderly people with frailty and co-morbidities, who are at the highest risk to develop severe forms of COVID-19, were either not included or underrepresented in these studies. Since immunosenescence may be detrimental to the functionality and breadth of vaccine-elicited humoral immune responses ${ }^{13}$, profiling of cross-neutralizing activity against VOCs of sera from fully vaccinated elderly people is of major public health interest. In the current work, we address this issue by comparing the neutralizing capacity of sera from Comirnaty COVID-19-vaccinated nursing home residents

\footnotetext{
${ }^{1}$ Institute for Integrative Systems Biology (I2SysBio), Universitat de Valencia-CSIC, 46980 Valencia, Spain. ${ }^{2}$ Microbiology Service, Clinic University Hospital, INCLIVA Health Research Institute, Valencia, Spain. ${ }^{3}$ Dirección de Atención Primaria, Departamento de Salud Clínico-Malvarrosa, Hospital Clínico Universitario de Valencia, Valencia, Spain. ${ }^{4}$ Dirección de Enfermería, Departamento de Salud Clínico-Malvarrosa, Hospital Clínico Universitario de Valencia, Valencia, Spain. ${ }^{5}$ Departamento de Salud València Clínico Malvarrosa, Comisión Departamental de Control de Residencias, Valencia, Spain. ${ }^{6}$ Department of Microbiology, School of Medicine, University of Valencia, Av. Blasco Ibáñez 17, 46010 Valencia, Spain. ${ }^{7}$ These authors contributed equally: Ron Geller and David Navarro. ${ }^{\circledR}$ email: david.navarro@uv.es
} 
with that of sera from younger and seemingly healthy vaccinated individuals and non-vaccinated individuals who recovered from severe COVID-19.

\section{Material and methods}

Study groups. In this observational retrospective study, three population groups were considered. Group 1 included 30 nursing home $(\mathrm{NH})$ residents with frailty and one or more co-morbidities (23 females), randomly selected from one $\mathrm{NH}$ affiliated to the Clínico-Malvarrosa Health Department, Valencia (Spain), aged a median of 90 years (range 64-100). Twenty-two were SARS-CoV-2 naïve, as judged by the absence of an historical records of having tested positive for SARS-CoV-2 RNA) or serological evidence of prior SARS-CoV-2 infection was available from any of these participants (all NHR tested negative for anti-N SARS-CoV-2 at the time of sampling), and 8 had contracted COVID-19 requiring hospitalization prior to receiving the first dose of the Comirnaty COVID-19 vaccine, as determined by previous records of positive RT-PCR results in nasopharyngeal specimens and the detection of SARS-CoV-2 N antibodies (at the time of sample collection). In all cases, the variant Wuhan/D614G was involved, as determined by whole-genome sequencing (not shown). All participants in this group were vaccinated on the same day and the sera were drawn 35 days after the second vaccine dose.

Group 2 comprised 18 healthy individuals (14 females) aged a median of 47.5 years (range 26-62), whose sera were drawn at a median of 15 days since the completion of the vaccination schedule (range 13-18). No molecular (historical records of having tested positive for SARS-CoV-2 RNA) or serological evidence of prior SARS-CoV-2 infection was available from any of these participants (all participants tested negative for anti- $\mathrm{N}$ SARS-CoV-2 at the time of sampling).

Group 3 included 19 unvaccinated individuals ( 7 females; median age, 63 years; range 40-80) who were infected with the Wuhan/D614G strain (determined by whole-genome sequencing; data not shown), required hospitalization either in clinical wards or intensive care units, and eventually recovered from severe COVID-19. Sera from these individuals were obtained at a median of 105 days (range 12-147) after molecular diagnosis of SARS-CoV-2 infection.

The study was approved by INCLIVA Research Ethics Committee, ethical statement number (2021/165). Given the retrospective nature of the analysis, the INCLIVA Research Ethics Committee exempted us from obtaining informed consent from patients. All methods were performed in accordance with Declaration of Helsinki and the Belmont Report guidelines and regulations.

Generation of SARS-CoV-2 with variant spikes. We introduced mutations in a mammalian expression vector encoding a codon-optimized SARS-CoV-2 S sequence from the Wuhan reference strain ${ }^{14}$. First, the D614G mutation was introduced by site-directed mutagenesis. Subsequently, additional site-directed mutagenesis and/or the cloning of synthetic fragments harboring the mutations (Gblocks, IDT) was performed to introduce all mutations using the NEBuilder HiFi DNA assembly mix (NEB). For the Beta variant (PANGO Lineage: B.1.351; mutations D80A, L241Del, L242Del, A243Del, E484K, N501Y, D614G, and A701V), a synthetic fragment encoding mutations D80A, L241Del, L242Del, A243Del, E484K, and N501Y was cloned, and the A701V mutation introduced by site-directed mutagenesis. For the Gamma variant (PANGO Lineage: P1 or B.1.1.28.1; mutations L18F, T20N, P26S, D138Y, R190S, K417T, E484K, N501Y, D614G, H655Y, T1027I, and V1176F), a synthetic fragment encoding mutations (L18F, T20N, P26S, D138Y, R190S, K417T, E484K, and N501Y) was cloned, and additional mutations H655Y, T1027I, and V1176F were inserted by site-directed mutagenesis. For the Delta variant (PANGO lineage: B.1.617.2; mutations T19R, del157/158, L452R, T478K, D614G, P681R, and $\mathrm{D} 950 \mathrm{~N}$ ) mutations were introduced by site-directed mutagenesis. Finally, for the Epsilon variant (PANGO lineages B.1.427/B.1.429; mutations S13I, W152C, L452R, D614G), a synthetic fragment encoding mutations S13I, W152C, and L452R was cloned. All plasmids were verified by Sanger sequencing.

Virus neutralization assay. The neutralization capacity of circulating antibodies (NtAb) against the SARS-CoV-2 S protein was assessed using a GFP-expressing vesicular stomatitis virus pseudotyped with different Spike variants as previously described ${ }^{15}$, but using A549-ACE2-TMPRSS2 cells (InvivoGen catalog code a549-hace2tps). All tests were done in duplicate using fivefold serum dilutions ranging from 1:20 to 1:62,500, with 1,000 focus forming units per well. Following $16 \mathrm{~h}$ of infection, the GFP signal in each well was quantified using a live-cell microscope (Incucyte S3, Sartorius). Background fluorescence from uninfected wells was subtracted from all infected well, and the GFP fluorescence in each antibody-treated dilution was standardized to the average fluorescence observed in mock-treated wells. Any value resulting in a relative GFP signal of $<0.001$ versus was assigned a value of 0.001 to eliminate negative values. Finally, the reciprocal antibody dilution resulting in $50 \%$ virus neutralization was calculated using the drc package (version 3.0-1) in $\mathrm{R}$ via a three-parameter log-logistic regression model (LL.3 model). For the Beta, Gamma, and Epsilon variants, all assays for the same serum were performed simultaneously with the Wuhan reference strain unless neutralization was repeated due to high variability. As the Delta variant was performed subsequently, we retested each serum was against both Delta and the Wuhan strains unless no neutralization of Wuhan was observed in the previous assays. Sera testing negative (undetectable) were arbitrarily ascribed a titer of $1 / 20$.

Quantitation of SARS-CoV-2 receptor binding domain-reactive antibodies. Total antibodies (IgG and IgM) against the SARS-CoV-2 S protein receptor-binding domain (RBD) were measured by Roche Elecsys electrochemiluminescence sandwich immunoassays (Roche Diagnostics, Pleasanton, CA, USA). The limit of detection of the assay is $0.4 \mathrm{IU} / \mathrm{ml}$ and its quantification range is between 0.8 and $250 \mathrm{IU} / \mathrm{ml}$. Plasma specimens were further diluted for antibody quantitation when appropriate. The assay is calibrated with the 

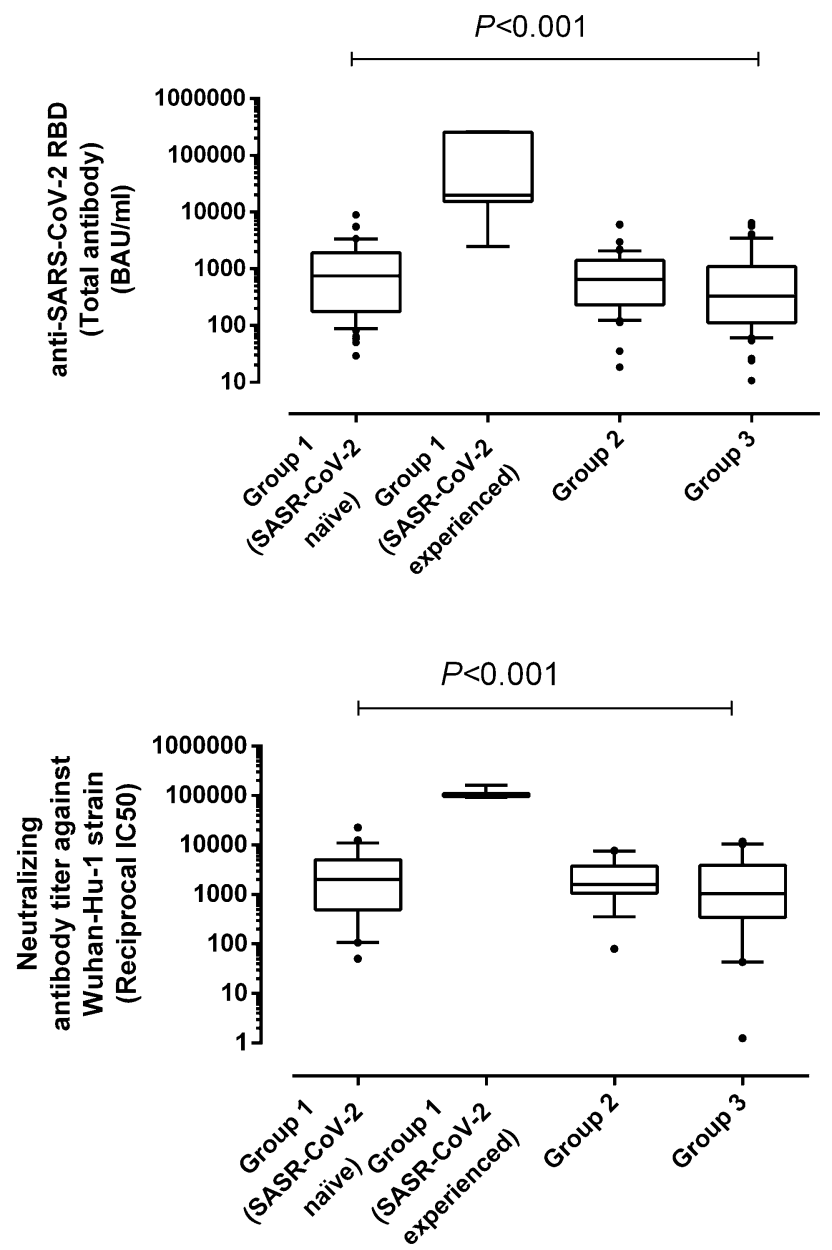

Figure 1. Box-Whisker plots depicting initial (Upper pannel) anti-SARS-CoV-2 RBD total antibody levels, as measured by the Roche Elecsys electrochemiluminescence sandwich immunoassays (Roche Diagnostics, Pleasanton, CA, USA) and neutralizing antibodies against SARS-CoV-2 Wuhan-Hu-1 variant (Lower pannel), in nursing home residents (Group 1), healthy individuals fully vaccinated with the Comirnaty vaccine (Group 2 ) and unvaccinated, COVID-19 recovered individuals that required hospitalization in internal medicine or intensive care units (Group 3). $P$ value for comparison across the three groups is shown.

first WHO International Standard and Reference Panel for anti-SARS-CoV-2 antibody ${ }^{16}$. Cryopreserved plasma $\left(-20^{\circ} \mathrm{C}\right)$ specimens were thawed and assayed in singlets.

Statistical methods. Geometric mean titers (GMTs) are reported throughout the study. Comparison of GMTs and medians across groups was carried out by the Student's t-test or the Mann-whitney U-test, as appropriate. Correlations between variables of interest were calculated by the Spearman Rank test. Frequency comparison across groups was performed using Fisher's exact test. Two-sided exact P values were reported. A P value $<0.05$ was considered statistically significant. The analyses were performed using SPSS version 20.0 (SPSS, Chicago, IL, USA).

\section{Results}

Detection of SARS-CoV-2-RBD and neutralizing antibodies in participants. Antibodies targeting the SARS-CoV-2 RBD were detectable in all but one (belonging to group 3) out of the 67 participants. Antibody levels across the study groups, shown in Fig. 1 (panel A), were found to differ significantly, with SARS-CoV2 -experienced nursing home residents displaying significantly higher levels as compared to that in SARS-CoV-2 naïve and participants from the other two study groups. In contrast, anti-RBD antibody levels were comparable across SARS-CoV-2 naïve, and individuals in groups 2 and $3(P \geq 0.5)$. Similarly, using a pseudotyped vesicular stomatitis virus system, NtAb against the ancestral variant were detected in all but one participants (belonging group 3, who also lacked anti-RBD antibodies). NtAb levels against the Wuhan-Hu-1 variant were significantly higher in SARS-CoV-2-experienced than in SARS-CoV-2 naive, and participants in groups 2 and 3 participants (Fig. 1, panel B). Measurable NtAb against the different SARS-CoV-2 VOCs included in the study were found at variable frequencies depending upon the study group and the virus variant considered (Table 1). In all groups except that corresponding to SARS-CoV-2-experienced nursing home residents, in which all individuals had 


\begin{tabular}{|l|l|l|l|l|}
\hline \multirow{2}{*}{} & \multicolumn{3}{|l|}{$\begin{array}{l}\text { Number of participants with detectable neutralizing antibodies/total } \\
\text { participants (\%) }\end{array}$} \\
\cline { 2 - 5 } & Group 1 & & \\
\cline { 2 - 5 } SARS-CoV-2 variant & SARS-CoV-2 nä̈ve & SARS-CoV-2 experienced & Group 2 & Group 3 \\
\hline Ancestral (Wuhan-Hu-1) & $22 / 22(100)$ & $8 / 8(100)$ & $18 / 18(100)$ & $18 / 19(94.7)$ \\
\hline Beta & $16 / 22(72.7)$ & $8 / 8(100)$ & $16 / 18(88.9)$ & $16 / 19(84.2)$ \\
\hline Gamma & $19 / 22(86.3)$ & $8 / 8(100)$ & $17 / 18(94.4)$ & $17 / 19(89.5)$ \\
\hline Delta & $20 / 22(90.9)$ & $8 / 8(100)$ & $18 / 18(100)$ & $17 / 19(89.5)$ \\
\hline Epsilon & $20 / 22(90.9)$ & $8 / 8(100)$ & $17 / 18(94.4)$ & $17 / 19(89.5)$ \\
\hline
\end{tabular}

Table 1. Detectable neutralizing antibodies against different SARS-CoV-2 variants across study groups. ${ }^{a}$ Group 1, nursing home residents fully vaccinated with the Comirnaty vaccine; Group 2, healthy individuals fully vaccinated with the Comirnaty vaccine; Group 3, unvaccinated, COVID-19 recovered individuals that required hospitalization in internal medicine or intensive care units.

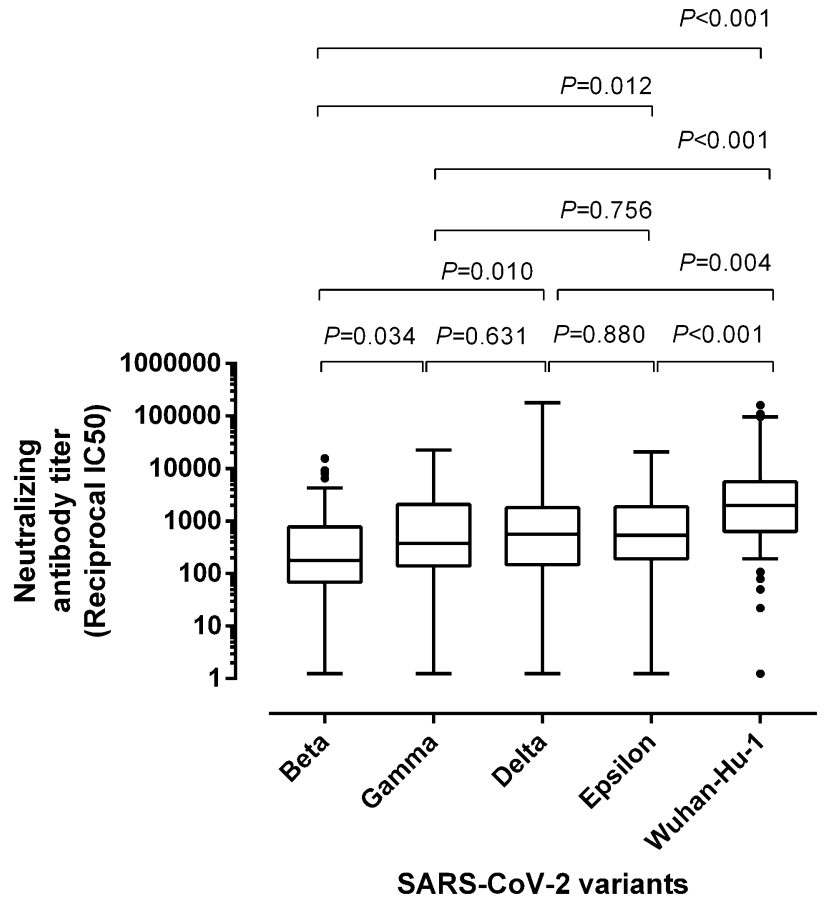

Figure 2. Box-whisker plots depicting neutralizing antibody titers against the SARS-CoV-2 ancestral strain (Wuhan-Hu-1) and variants of concern Beta, Gamma, Delta and Epsilon in sera form participants. $P$ values for pairwise comparisons are shown.

detectable $\mathrm{NtAb}$ against all variants, some participants lacked NtAb against one or more VOCs, most notably the Beta variant (15-20\%). As for vaccinated individuals (groups 1 and 2), no significant differences in the detection rate of $\mathrm{NtAb}$ for any SARS-CoV-2 VOCs were found $(P \geq 0.5)$ (Table 1$)$.

Antibody neutralizing titers against SARS-CoV-2 S variants across study groups. Overall, all VOCs were neutralized to a lesser extent than the ancestral strain (Fig. 2) by sera belonging to participants in the different study groups. Specifically, serum NtAb titers were lowest against the Beta variant followed by Gamma, Delta and Epsilon variants, although wide ranges were observed for all variants. This pattern was shared by all study groups except for SARS-CoV-2-experienced participants in group 1, who displayed a remarkable NtAb activity against the Delta variant, and group 2 individuals who overall displayed an slightly decresased neutralizing activity against Epsilon as compared to the Delta variant (Table 2 and Supplementary Fig. 1). Fold change reduction in NtAb titers relative to the ancestral variant was overall greatest for the Beta variant (6.7-19.4) followed by Gamma (4.8-16.0), Epsilon (2.9-13.4), and Delta (3.5-6.5) variants. The decrease in NtAb titers for SARS-CoV-2 Gamma variant was not significantly different across comparison groups (Fig. 3); nevertheless, there were differences across comparison groups for the remaining VOCs; specifically, overall, group 3 participants exhibited a less prominent fold-change decrease in NtAb titers against the Beta variant, SARS-CoV-2-ex- 


\begin{tabular}{|c|c|c|c|c|c|}
\hline \multirow[b]{3}{*}{ SARS-CoV-2 variant } & \multirow[b]{3}{*}{ Parameter } & \multicolumn{4}{|l|}{ Study groups $^{\mathrm{a}}$} \\
\hline & & \multicolumn{2}{|l|}{ Group 1} & \multirow[b]{2}{*}{ Group 2} & \multirow[b]{2}{*}{ Group 3} \\
\hline & & SARS-CoV-2 Naïve & SARS-CoV-2 experienced & & \\
\hline \multirow[b]{2}{*}{ Beta } & GMT (SD) & $73.2(612)$ & $6,172(5,078)$ & $124(316)$ & $122(999)$ \\
\hline & $\begin{array}{l}\begin{array}{l}\text { Mean fold change reduction } \\
\text { of NtAb titer (CI 95\%) }\end{array} \\
\end{array}$ & $19.4(147.8)$ & $17.4(27.5)$ & $13.1(6.9)$ & $6.7(84.9)$ \\
\hline \multirow[t]{2}{*}{ Gamma } & GMT (SD) & $241.7(2,013)$ & $14,641(6,777)$ & $256(435)$ & $225(1607)$ \\
\hline & $\begin{array}{l}\text { Mean fold change reduction } \\
\text { of NtAb titer (CI 95\%) }\end{array}$ & $5.88(10.4)$ & $7.35(16.0)$ & $6.3(6.6)$ & $3.6(4.8)$ \\
\hline \multirow[t]{2}{*}{ Delta } & GMT (SD) & $216.6(3,274)$ & $77,157(79,273)$ & $543(1,138)$ & $231(1,162)$ \\
\hline & $\begin{array}{l}\text { Mean fold change reduction } \\
\text { of NtAb titer (CI 95\%) }\end{array}$ & $6.57(14.7)$ & $1.39(1.62)$ & $3.0(0.6)$ & $3.5(4.1)$ \\
\hline \multirow[t]{2}{*}{ Epsilon } & GMT (SD) & $330(4,309)$ & $8,047(7,189)$ & $261(324)$ & $283(1,869)$ \\
\hline & $\begin{array}{l}\text { Mean fold change reduction } \\
\text { of NtAb titer (CI 95\%) }\end{array}$ & $4.3(8.1)$ & $13.4(15.4)$ & $6.2(6.6)$ & $2.9(2.2)$ \\
\hline (Wuhan-Hu-1) & GMT (SD) & $1,423(5,173)$ & $107,541(20,934)$ & $1,622(2,259)$ & $442(2,083)$ \\
\hline
\end{tabular}

Table 2. Serum neutralizing antibody titers against SARS-CoV-2 variants across study groups. CI confidence interval, GMT geometric mean titer, $N t A b$ neutralizing antibodies, $S D$ standard deviation. ${ }^{\mathrm{a}}$ Group 1 , nursing home residents fully vaccinated with the Comirnaty vaccine; Group 2, healthy individuals fully vaccinated with the Comirnaty vaccine; Group 3, unvaccinated, COVID-19 recovered individuals that required hospitalization in internal medicine or intensive care units. ${ }^{b}$ Relative to the NtAb titer against WA1/2020.

Beta

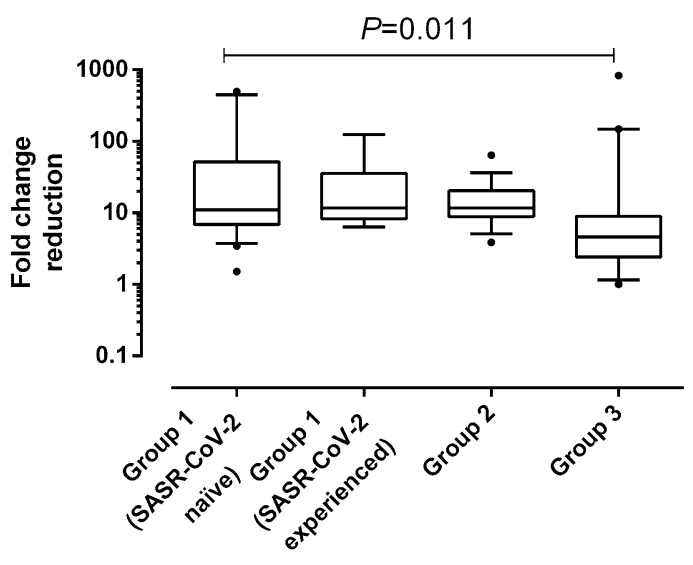

Delta

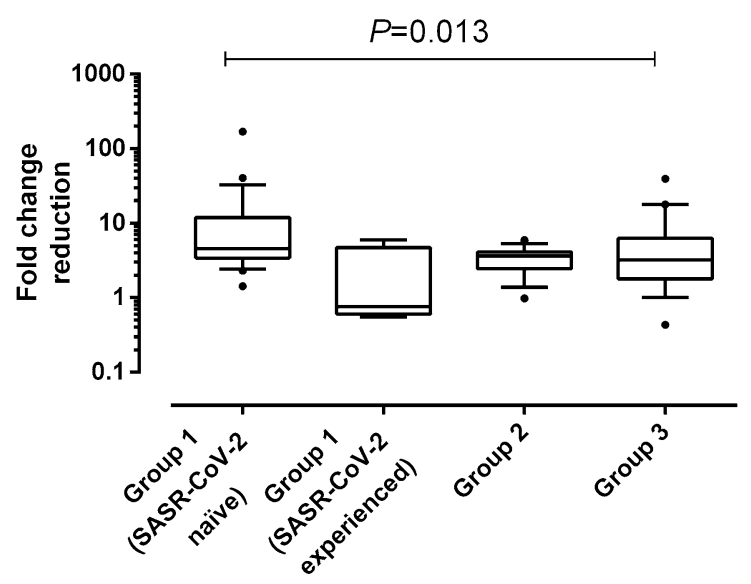

Gamma

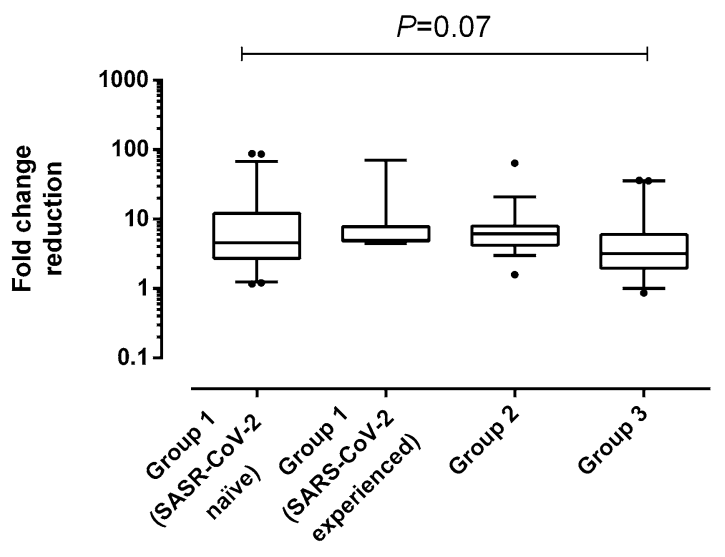

Epsilon

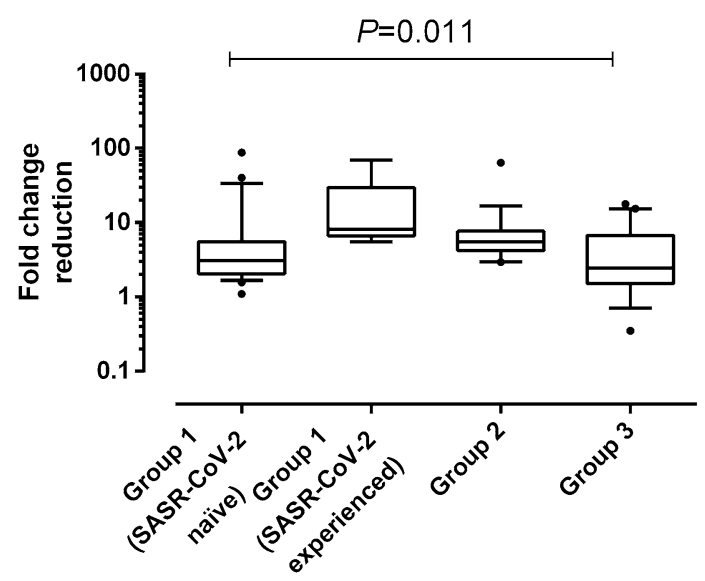

Figure 3. Box-whisker plots depicting fold change reduction in neutralizing antibody titers against SARS$\mathrm{CoV}-2$ variants of concern as compared to the ancestral strain as a function of the study group considered. $P$ value for comparison across the three groups is shown. 

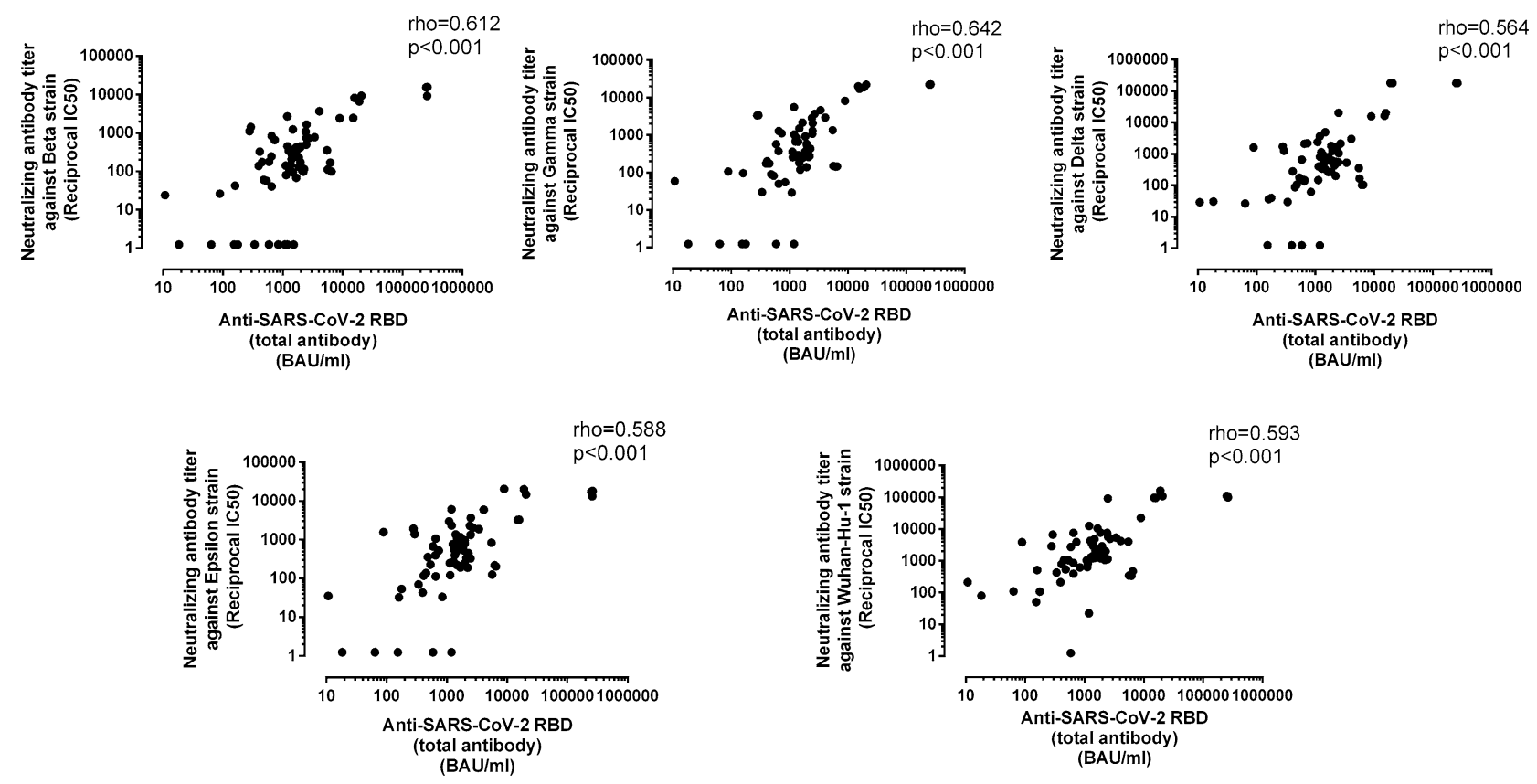

Figure 4. Overall correlation between neutralizing antibody titers and anti-SARS-CoV-2 RBD total antibody levels, as measured by the Roche Elecsys electrochemiluminescence sandwich immunoassays (Roche Diagnostics, Pleasanton, CA, USA), according to the SARS-CoV-2 S-variant. Rho and $P$ values are shown.

perienced nursing home residents did so against the Delta variant, whereas the latter individuals also displayed a more marked decrease against the Epsilon variant.

Correlation between SARS-CoV-2 neutralizing and anti-RBD antibodies. The degree of correlation between levels of SARS-CoV-2 antibodies neutralizing SARS-CoV-2 VOCs and total anti-RBD antibodies across the different population groups was examined next. Overall, the correlation between these two parameters was rather similar for the ancestral strain and and all VOCs and moderate in all cases (Fig. 4). Of interest, this pattern was rather similar for both the vaccinated participants from nursing home residents, irrespective of their SARS-CoV-2 infection status prior to vaccination, and healthy controls; nevertheless, a slightly lower correration between anti-RBD antibodies and $\mathrm{NtAb}$ levels was noticed for SARS-CoV-2-experienced nursing home residents (Supplementary Table 1).

\section{Discussion}

Reduced neutralization of VOCs as compared to the ancestral Wuhan-Hu-1 strain has been observed in sera from individuals that were fully vaccinated against SARS-CoV-2 or have recovered from infection by either the ancestral Wuhan or the Wuhan/D164G SARS-CoV-2 strains ${ }^{5-12,17,18}$. This has been noticed in both the full SARS-CoV-2 virus or pseudotyped virus systems and is most notable for the Beta variant. As for recipients of the Comirnaty COVID-19 vaccine, this observation has been mostly made in vaccine clinical trials participants and seemingly healthy health workers ${ }^{5-12}$. Here, using S-pseudotyped SARS-CoV-2 variants, we extend this finding to include elderly nursing home residents, both in SARS-CoV-2 naïve participants and those infected by the Wuhan/D614G variant prior to vaccination. In effect, 1 month after the second vaccine dose, sera from fully vaccinated SARS-CoV-2 naïve and experienced nursing home residents retained the ability to neutralize VOCs in most and all cases, respectively, but did so to a lesser extent than the ancestral strain, especially in the case of the Beta variant. Importantly, reduction in NtAb activity against VOCs in this population group was not significantly different from that seen in healthy fully-vaccinated controls (in sera obtained a median of two weeks after vaccination); in fact, it was significantly less pronounced for the Delta variant in SARS-CoV-2-experienced individuals. Nevertheless, unvaccinated individuals who had recovered from severe COVID-19 diagnosed within a median time of 3 months had a less marked fold-change reduction in NtAb levels against the Beta and Epsilon variants. Leaving aside participants who remained unvaccinated and naturally contracted the infection, which are not strictly comparable to vaccinees, our data strongly support the idea that older age, frailty, and concurrence of co-morbidities in fully-vaccinated individuals had no major impact on the serum $\mathrm{NtAb}$ activity profile against VOCs, including Delta variant that has been predominant in many countries until the emergence of the Omicron variant. The reduction in NtAb activity for all VOCs relative to that observed for the Wuhan-Hu-1 reference strain (fold-change reduction in $\mathrm{NtAb}$ activity) was higher for all VOCs compared to other studies, especially the Beta variant. For example, using a pseudotyped virus system, Liu et al. ${ }^{5-7}$ reported a 2.7- and 1.4fold reduction in the $\mathrm{NtAb}$ activity against Beta and Delta variants, respectively, for sera from participants in the Comirnaty COVID-19 vaccine the trial, who were seemingly comparable to our healthy vaccinated controls (13.1 and 3.0 fold reduction, respectively). In turn, using live virus isolates in microneutralization assays, Lustig and 
colleagues $^{11}$ found fold change reduction in NtAb titers of 10.4, 2.3, and 2.1 against the Beta, Gamma, and Delta variants compared with the ancestral virus in health care workers fully vaccinated with the Comirnaty COVID-19 vaccine. Comparison of fold change reduction figures across studies is not straightforward, due not only to differences between population group characteristics, especially in terms of age, sex, comorbidities, and SARS-CoV-2 infection status prior to vaccination, but also to differences in the pseudotyped virus platforms, the chosen $S$ sequences used to represent each variant or the use of live viruses. Despite these differences, a similar pattern of $\mathrm{NtAb}$ activity against VOCs can be derived from these studies as well as our current work, with the Beta variant being less efficiently neutralized than the ancestral strain, followed by the Gamma, Delta and Epsilon variants.

Levels of total antibodies binding SARS-CoV-2 RBD as quantitated by the Roche Elecsys electrochemiluminescence sandwich immunoassays strongly correlate with NtAb titers against the ancestral SARS-CoV-2 strain ${ }^{19,20}$. Here we extended this observation to those against Beta, Gamma, Delta, and Epsilon variants, suggesting that SARS-CoV-2 RBD antibody levels measured by this assay may be a reliable proxy for serum NtAb activity against these VOCs, regardless of the vaccinated population group considered.

The relatively small sample size could be considered a limitation of the current study; this particularly concerned the subgroup of SARS-CoV-2-experienced nursing home residents, so that conclusions drawn regarding this population group should be taken with caution and warrants further investigations. Also, a limitation inherent to the use of S-pseudotyped viruses is the inability to assess the impact of variations in other viral genes outside of the $S$ protein that could affect sera neutralization.

In summary, herein we show that neutralizing activity of sera against SARS-CoV-2 variants carrying critical escape mutations in the $\mathrm{S}$ gene is decreased relative to the ancestral strain in both qualitative and quantitative terms in nursing home residents recently vaccinated with the Comirnaty COVID-19 vaccine, most notably in those who were SARS-CoV-2 naïve prior to vaccination. Nevertheless, the fold reduction in NtAb activity in this population group was not significantly different from that seen in vaccinated younger controls. Nevertheless, the degree to which reduced neutralization activity of the different VOCs, and especially the Delta variant, impact infection and/or disease in vaccinated nursing home residents translate into diminished effective protection is currently under investigation.

Received: 28 October 2021; Accepted: 25 February 2022

Published online: 08 March 2022

\section{References}

1. Smith, E. C., Blanc, H., Surdel, M. C., Vignuzzi, M. \& Denison, M. R. Coronaviruses lacking exoribonuclease activity are susceptible to lethal mutagenesis: Evidence for proofreading and potential therapeutics. PLoS Pathog. 9, e1003565. https://doi.org/10.1371/ journal.ppat.1003565 (2013).

2. Vkovski, P., Kratzel, A., Steiner, S., Stalder, H. \& Thiel, V. Coronavirus biology and replication: Implications for SARS-CoV-2. Nat. Rev. Microbiol. 19, 155-170. https://doi.org/10.1038/s41579-020-00468-6 (2021).

3. Boehm, E. et al. Novel SARS-CoV-2 variants: The pandemics within the pandemic. Clin. Microbiol. Infect. 27, 1109-1117. https:// doi.org/10.1016/j.cmi.2021.05.022 (2021).

4. Sahin, U. et al. COVID-19 vaccine BNT162b1 elicits human antibody and TH1 T cell responses. Nature 586, 594-599. https://doi. org/10.1038/s41586-020-2814-7 (2020).

5. Liu, Y. et al. Neutralizing activity of BNT162b2-elicited serum. N. Engl. J. Med. 384, 1466-1468. https://doi.org/10.1056/NEJMc 2102017 (2021).

6. Liu, Y. et al. BNT162b2-elicited neutralization against new SARS-CoV-2 spike variants. N. Engl. J. Med. 385, 472-474. https://doi. org/10.1038/s41586-021-03693-y (2021).

7. Liu, J. et al. BNT162b2-elicited neutralization of B.1.617 and other SARS-CoV-2 variants. Nature 596, 273-275. https://doi.org/ 10.1038/s41586-021-03693-y (2021).

8. Xie, X. et al. Neutralization of SARS-CoV-2 spike 69/70 deletion, E484K and N501Y variants by BNT162b2 vaccine-elicited sera. Nat. Med. 27, 620-621. https://doi.org/10.1038/s41591-021-01270-4 (2021).

9. Jalkanen, P. et al. COVID-19 mRNA vaccine induced antibody responses against three SARS-CoV-2 variants. Nat. Commun. 12, 3991. https://doi.org/10.1038/s41467-021-24285-4 (2021).

10. Liu, C. et al. Reduced neutralization of SARS-CoV-2 B.1.617 by vaccine and convalescent serum. Cell 184, 4220-4236. https://doi. org/10.1016/j.cell.2021.06.020 (2021).

11. Lustig, Y. et al. Neutralising capacity against Delta (B.1.617.2) and other variants of concern following Comirnaty (BNT162b2, BioNTech/Pfizer) vaccination in health care workers. Israel. Euro Surveill. 26, 2100557. https://doi.org/10.2807/1560-7917.ES. 2021.26.26.2100557 (2021).

12. Wang, Z. et al. mRNA vaccine-elicited antibodies to SARS-CoV-2 and circulating variants. Nature 592, 616-622. https://doi.org/ 10.1038/s41586-021-03324-6 (2021).

13. Jergović, M., Coplen, C. P., Uhrlaub, J. L. \& Nikolich-Žugich, J. Immune response to COVID-19 in older adults. J. Heart Lung Transplant. https://doi.org/10.1016/j.healun.2021.04.017 (2021).

14. Hoffmann, M. et al. SARS-CoV-2 cell entry depends on ACE2 and TMPRSS2 and is blocked by a clinically proven protease inhibitor. Cell 181, 271-280.e8. https://doi.org/10.1016/j.cell.2020.02.052 (2020).

15. Gozalbo-Rovira, R. et al. SARS-CoV-2 antibodies, serum inflammatory biomarkers and clinical severity of hospitalized COVID-19 patients. J. Clin. Virol. 131, 104611. https://doi.org/10.1016/j.jcv.2020.104611 (2020).

16. Mattiuzzo G, et al. Establishment of the WHO International Standard and Reference Panel for anti-SARS-CoV-2 antibody. WHO/ BS/2020.2403, December 10 (2020).

17. Weisblum, Y. et al. Escape from neutralizing antibodies by SARS-CoV-2 spike protein variants. Elife 9, e61312 (2020).

18. Barros-Martins, J. et al. Immune responses against SARS-CoV-2 variants after heterologous and homologous ChAdOx1 nCoV-19/ BNT162b2 vaccination. Nat. Med. 27, 1525-1529. https://doi.org/10.1038/s41591-021-01449-9 (2021).

19. Higgins, V., Fabros, A. \& Kulasingam, V. Quantitative measurement of anti-SARS-CoV-2 antibodies: Analytical and clinical evaluation. J. Clin. Microbiol. 59, e03149-e3220. https://doi.org/10.1128/JCM.03149-20 (2021).

20. Poljak, M., Oštrbenk Valenčak, A., Štamol, T. \& Seme, K. Head-to-head comparison of two rapid high-throughput automated electrochemiluminescence immunoassays targeting total antibodies to the SARS-CoV-2 nucleoprotein and spike protein receptor binding domain. J. Clin. Virol. 137, 104784. https://doi.org/10.1016/j.jcv.2021.104784 (2021). 


\section{Acknowledgements}

We are grateful to all personnel who work at nursing home residences affiliated with the Clínico-Malvarrosa Health Department and at Clinic University Hospital, in particular to those at the Microbiology laboratory, for their commitment in the fight against COVID-19. Ignacio Torres holds a Río Hortega Contract (CM20/00090) from the Carlos III Health Institute. Eliseo Albert holds a Juan Rodés Contract (JR20/00011) from the Carlos III Health Institute. Estela Giménez holds a Juan Rodés Contract (JR18/00053) from the Carlos III Health Institute. Ron Geller holds a Ramon y Cajal Fellowship from the Spanish Ministry of Economy and Competitiveness (RYC-2015-17517).

\section{Author contributions}

B.S.-S., E.A., J.Z., I.T., and E.G.: Methodology and data validation. P.B., M.J.B., C.R.: in charge of implementing public health policies to combat SARS-CoV-2 epidemic at nursing home residences affiliated to the ClínicoMalvarrosa Health Department. R.G. and D.N.: Conceptualization, supervision, writing the original draft. All authors reviewed the original draft.

\section{Funding}

This research work was supported by a grant from the European Commission- NextGeneration EU.

\section{Competing interests}

The authors declare no competing interests.

\section{Additional information}

Supplementary Information The online version contains supplementary material available at https:/doi.org/ 10.1038/s41598-022-07849-2.

Correspondence and requests for materials should be addressed to D.N.

Reprints and permissions information is available at www.nature.com/reprints.

Publisher's note Springer Nature remains neutral with regard to jurisdictional claims in published maps and institutional affiliations.

(c) (i) Open Access This article is licensed under a Creative Commons Attribution 4.0 International License, which permits use, sharing, adaptation, distribution and reproduction in any medium or format, as long as you give appropriate credit to the original author(s) and the source, provide a link to the Creative Commons licence, and indicate if changes were made. The images or other third party material in this article are included in the article's Creative Commons licence, unless indicated otherwise in a credit line to the material. If material is not included in the article's Creative Commons licence and your intended use is not permitted by statutory regulation or exceeds the permitted use, you will need to obtain permission directly from the copyright holder. To view a copy of this licence, visit http://creativecommons.org/licenses/by/4.0/.

(C) The Author(s) 2022 